\title{
Analysis of the Impact of Climatic Conditions on Floral Transformation in Hydrangea macrophylla 'Leuchtfeuer'
}

\author{
Gilles Galopin ${ }^{1}$ \\ Agrocampus Ouest, UMR SAGAH A 462, 2 rue Le Nôtre, 49045 Angers, \\ France
}

\author{
Laurent Crespel and Jean C. Mauget \\ Agrocampus Ouest, UMR GenHort A 1259, 2 rue Le Nôtre, 49045 Angers, \\ France
}

Philippe Morel
INRA, UMR SAGAH A 462, 42 rue Georges Morel, 49071 Beaucouzé, France

Additional index words. floral induction, morphogenesis, organogenesis

\begin{abstract}
Hydrangea macrophylla is a horticultural plant of considerable commercial interest that has been widely studied with the aim to more effectively control the different stages of its development during production. However, although floral transformation is a key factor underlying the commercial quality of the product, it remains difficult to control despite these efforts. The floral transformation sequence consists of three successive phases: floral induction $\left(B_{1}\right)$, floral evocation $\left(B_{2}\right)$, and floral organogenesis $\left(B_{3}\right)$. The first is a phase of vegetative organogenesis without elongation leading to the formation of a bud composed of eight phytomer primordia under inductive climatic conditions. This work shows that climatic conditions favorable to floral transformation must be continuously applied without interruption throughout phase $B_{1}$ to ensure the formation of the floral bud in Hydrangea macrophylla 'Leuchtfeuer'. In the opposite case, floral transformation is stopped and vegetative growth begins once again.
\end{abstract}

Hydrangea macrophylla is an ornamental horticultural plant grown in pots for indoor and outdoor use. The commercial quality of potted plants is defined by the number of flowering axes, their morphometric homogeneity, and the synchronization of flower bloom. The flowering axis develops over 2 years of growth (Galopin, 1995). Continuous plant growth over the first year ensures the formation of the stem. It is followed by floral transformation that is morphologically identifiable by the formation of a mixed bud composed of scale leaves, phytomer primordia, and floral primordia (Galopin et al., 2008; Uemachi and Nishio, 2000; Zhou and Hara, 1988). This floral transformation occurs at the end of the summer in temperate climates. It is followed by the onset of endodormancy of the bud that requires cooler temperatures to restore growth capability (Fuchigami and Wisniewski, 1997; Wallerstein, 1981). The second year, terminal flowering is ensured by the elongation of all of the vegetative and floral organs preformed in the mixed bud.

When plants are in production, we frequently observe either heterogeneous flower-

\footnotetext{
Received for publication 22 June 2011. Accepted for publication 12 Aug. 2011.

We thank Michel Laffaire and Rémy Gardet for their technical assistance and Gail Wagman for the English translation of the text.

${ }^{1}$ To whom reprint requests should be addressed; e-mail gilles.galopin@agrocampus-ouest.fr.
}

ing axes at the top of the plant or axes without inflorescences that totally degrade the quality of the product. Improved knowledge of the morphogenetic processes involved in floral transformation and, in particular, inductive environmental factors would make it possible to avoid these losses in quality.

Many researchers have attempted to identify the climatic factors favorable to floral induction. Thus, temperatures below $18^{\circ} \mathrm{C}$ (Bailey and Weiler, 1984; Post, 1942) and a short photoperiod of less than $12 \mathrm{~h}$ (Guo et al., 1995; Morita et al., 1980; Shanks et al., 1986) are favorable to floral transformation. Despite some interesting results, this research has not yet made it possible to control floral transformation during production. More recent studies have led to a detailed and chronological description of floral transformation in $H$. macrophylla (Fig. 1). The floral transformation sequence consists of three successive phases: 1) floral induction with the formation of the bud composed of eight vegetative preformed primordia $\left(\mathrm{B}_{1}\right) ; 2$ ) floral evocation with an increase in the size of the meristem and an activation of the entire apical zone $\left(\mathrm{B}_{2}\right)$; and 3 ) floral organogenesis with a differentiation of floral primordia $\left(\mathrm{B}_{3}\right)$ (Galopin et al., 2008, 2010).

The floral induction phase $\left(\mathrm{B}_{1}\right)$ is defined as the transition from the capacity of the meristem to produce leaves to its capacity to produce flowers after the perception of a climatic (O'Hare, 2004) or agronomic (Chaikiattiyos et al., 1994) signal. The research presented here focuses on observations of the impact of the transition from climatic conditions favorable to floral induction to those favorable to vegetative growth in $H$. macrophylla during this phase $\left(B_{1}\right)$. This change in climatic conditions can be found during production, which is why it is important to assess its consequences on plant development.

\section{Materials and Methods}

Plant material. The experiment was carried out on Hydrangea macrophylla 'Leuchtfeuer'. This deep pink genotype with globular inflorescences has a wide phenotypic variability because it is used as an ornamental plant outdoors, indoors in pots, and for cut flowers. Young plants were obtained from juvenile cuttings, cloned, and produced by mother microplant culture (Galopin et al., 1996). Cuttings consisted of two pairs of leaves left to root in 33-mm-diameter alveolated sheets filled with pre-moistened perlite and placed on subirrigation tables in a greenhouse (air temperature: $21 \pm 2{ }^{\circ} \mathrm{C}$; photoperiod: $16 \mathrm{~h}$; relative humidity greater than $85 \%$ ). Young plants were repotted 1 month after the initial cutting.

Experimental design. The experiment took place in Angers, France (lat. $47^{\circ} 33^{\prime} 37^{\prime \prime} \mathrm{N}$, long. $00^{\circ} 18^{\prime} 44^{\prime \prime} \mathrm{W}$, altitude $59 \mathrm{~m}$ ). The young plants were repotted on 23 Oct. 2006 in 7-cmdiameter pots in a substrate consisting of peat $(60 \%)$ and perlite $(40 \%)$ and placed in a greenhouse. The minimum air temperature was $16^{\circ} \mathrm{C}$ with ventilation when the air temperature reached $18{ }^{\circ} \mathrm{C}$. The photoperiod was naturally short and decreased over time from $10 \mathrm{~h}$ to $8 \mathrm{~h}$. The average quantity of light energy in the photosynthetically active radiation $(P A R)$ per day was $1200,1000,950,950$, and $820 \mathrm{~kJ}$ for the 5 months from October to February, respectively. The sample consisted of 500 plants. The control plants (T0) were maintained in the greenhouse throughout the experiment. The plants of treatments $\mathrm{T} 1, \mathrm{~T} 2$, $\mathrm{T} 3, \mathrm{~T} 4$, and $\mathrm{T} 5$ were transferred to a climatic chamber when the number of phytomer primordia in the bud was equal to four, five, six, seven, and eight, respectively. The climatic conditions in the chamber were favorable to vegetative growth with a photoperiod of $16 \mathrm{~h}$, a thermoperiod of $22{ }^{\circ} \mathrm{C}$ during the day and $20{ }^{\circ} \mathrm{C}$ at night, and a relative humidity greater than $80 \%$. The quantity of light energy in the $P A R$ was $955 \mathrm{~kJ}$ with a photon flux density of $70 \mu \mathrm{mol} \cdot \mathrm{m}^{-2} \cdot \mathrm{s}^{-1}$ using fluorescent tubes (Sylvania T8, F58W). The plants were irrigated by localized fertigation with a nutrient solution consisting of the following major elements in $\mathrm{mol} \cdot \mathrm{m}^{-3}: 1.7 \mathrm{~N}-0.38 \mathrm{P}-2.4 \mathrm{~K}-1 \mathrm{Ca}-$ $0.4 \mathrm{Mg}$ ( $\mathrm{pH} 6.5$ ).

Measurements and expression of results. Analysis of vegetative and floral organogenesis was carried out on the basis of destructive observations and measurements made once a week on 12 plants for $\mathrm{T} 0$ and on six plants for $\mathrm{T} 1, \mathrm{~T} 2, \mathrm{~T} 3, \mathrm{~T} 4$, and T5. It was carried out on three morphological components: the number of visible phytomers, the number of phytomer primordia in the bud, and the floral stage. The phytomer consists of an internode, a node, 
two axillary buds, and a pair of leaves (Barlow, 1989; White, 1979). The floral stage was ranked from 1 to 7 in relation to the stage of organogenesis reached $(1=$ vegetative stage; $2=$ one inflorescent meristematic dome; 3 = three meristematic domes; $4=$ nine meristematic domes; $5=a$ large number of inflorescent meristematic domes; $6=$ differentiation of floral primordia; $7=$ differentiation of floral organs) (Galopin et al., 2008). These measurements allowed us to calculate the time necessary for true leaf initiation, known as the true plastochron, which corresponds to the time between the formation of the two successive phytomer primordia by the meristem and is calculated using a linear regression curve.

Statistical analyses were made on the basis of the calculation of the mean with a confidence interval of $5 \%$.

\section{Results}

Evolution of vegetative and floral organogenesis in the mixed bud under climatic conditions favorable to floral transformation (T0). Apical organogenesis is characterized by the evolution of the number of phytomer primordia and the floral stage in the mixed bud (Fig. 2). The number of phytomer primordia in the vegetative bud was $3 \pm 0.0$ on Day 0 and increased up to Day 83 when it reached the value of $8.42 \pm 0.79$. From the first to the 27 th day, the true plastochron increased linearly according to the equation curve, $\mathrm{y}=0.1154 \mathrm{x}+3.1237\left(R^{2}=0.98 ; \mathrm{y}=\right.$ number of primordia; $\mathrm{x}=$ number of days) with an average value of $8.6 \mathrm{~d}$. From the $33 \mathrm{rd}$ to the 83rd day, the true plastochron increased according to the equation curve, $\mathrm{y}=0.0478 \mathrm{x}+$ $4.1115\left(R^{2}=0.87\right)$, with an average value of $20.9 \mathrm{~d}$. During this period, the number of visible phytomers remained stable and equal to

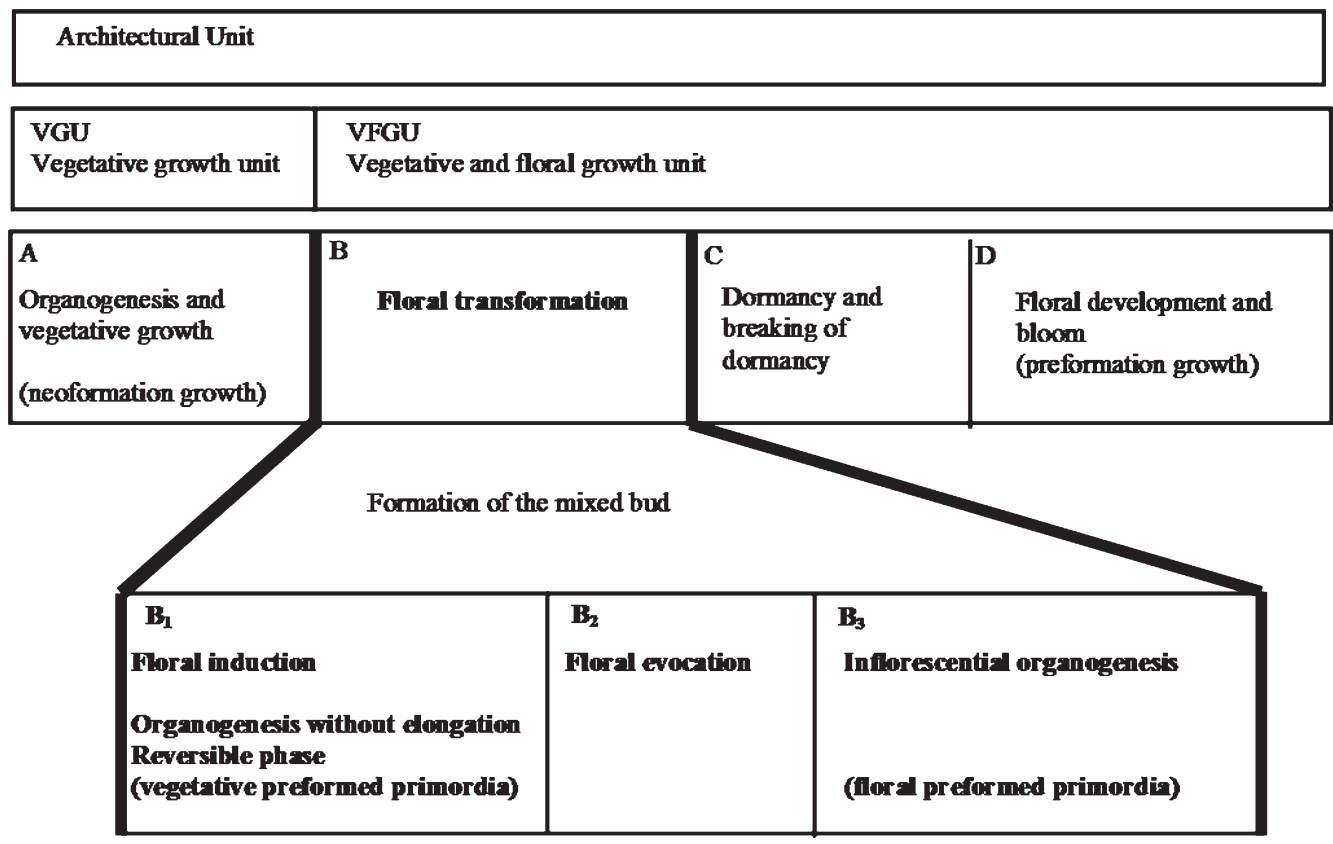

Fig. 1. The development sequences of the floral axis of Hydrangea macrophylla. The floral axis is composed of two morphogenesis units (VGU and VFGU) and four development sequences (A, B, C, D) (Galopin et al., 2010). The floral transformation sequence is composed of three phases $\left(B_{1}, B_{2}, B_{3}\right)$.

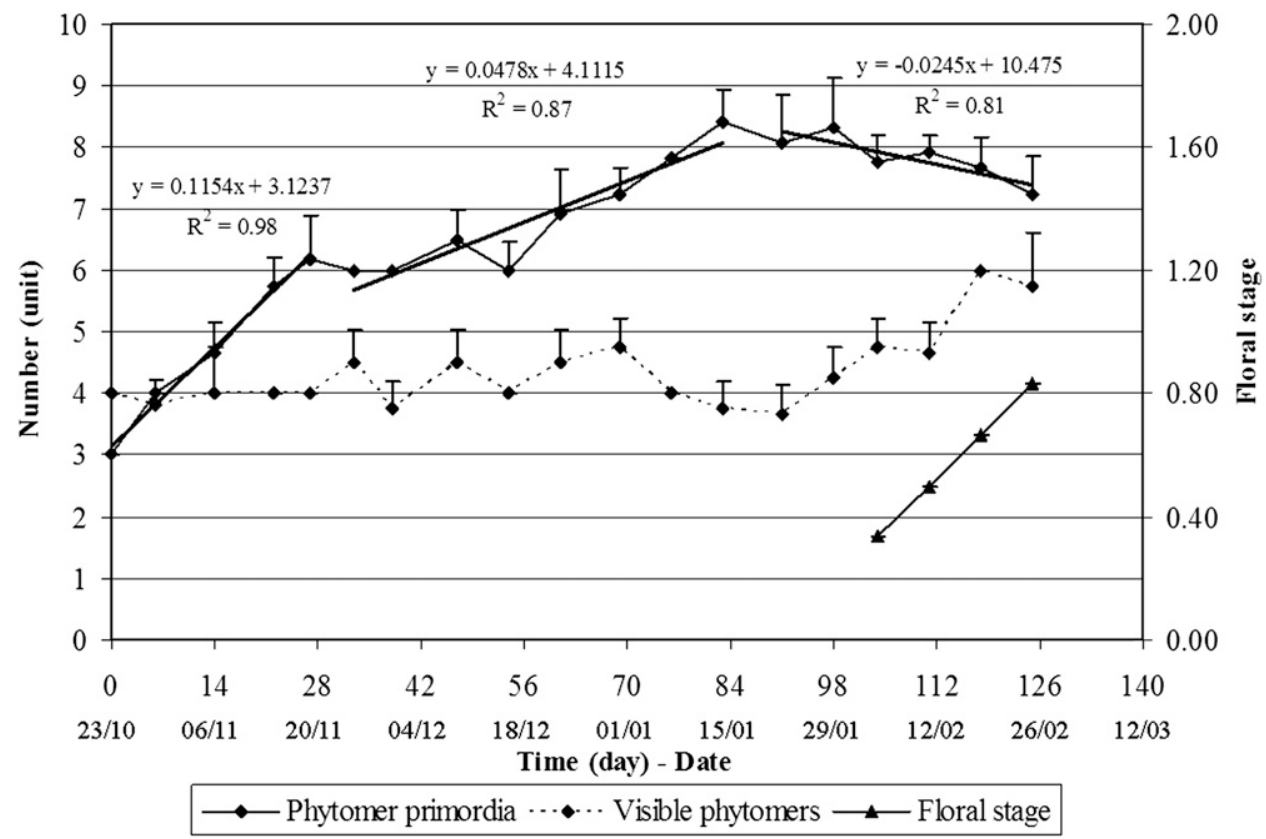

Fig. 2. Evolution of vegetative and floral organogenesis in the bud under climatic conditions favorable to floral transformation (T0). Number of phytomer primordia in the terminal bud; number of visible phytomers; floral stage. 
$4.12 \pm 0.33$. The end of vegetative organogenesis took place from the 91 st day to the 125 th day. Floral organogenesis began on the 104th day and evolved to the $0.86 \pm 0.58$ stage until the 125th day. This accumulation of leaves and floral primordia at the apical level led to the formation of a mixed bud.

Evolution of vegetative organogenesis in the bud under climatic conditions favorable to vegetative growth (T1 to T5). For treatment $\mathrm{T} 1$, the stem lengthened with an increase in the number of visible phytomers that corresponded to the growth of external phytomer primordia preformed in the bud (Fig. 3). Of the $3.83 \pm$ 0.41 phytomer primordia preformed in the bud, only the most external one lengthened; the other three formed a permanent primordia stock that was regularly renewed by meristematic activity. Growth then continued with continuous organogenesis and the regular emergence of new phytomers (Fig. 4).

For treatments T2, T3, T4 and T5, the same evolution was observed. The number of phytomer primordia preformed in the bud, observed at the time of the plant transition to conditions favorable to vegetative growth (Day
$0)$, was $4.83 \pm 0.41,6.17 \pm 0.41,7.17 \pm 0.41$, and $8.0 \pm 0.0$, respectively (Fig. 4). It then rapidly decreased to three for all of the treatments coupled with a proportional increase in the number of visible phytomers (Fig. 3).

\section{Discussion}

The quantitative and dynamic analysis of the organogenesis and growth of Hydrangea macrophylla requires a large number of measurements, some of which are destructive. Data quality was ensured by the homogeneity of

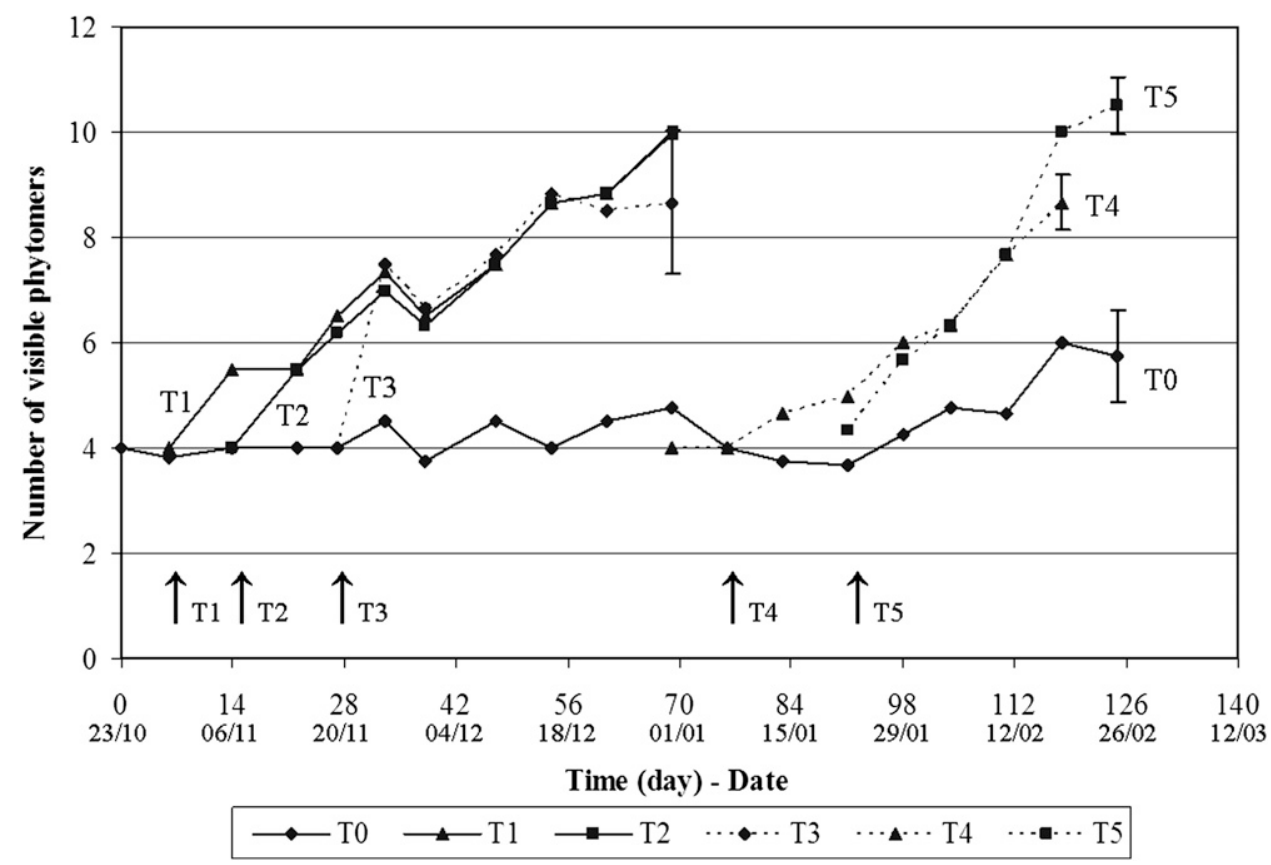

Fig. 3. Evolution of the number of visible phytomers for treatments T0 (control plants) and T1 to T5 as defined in the caption for Figure 4.

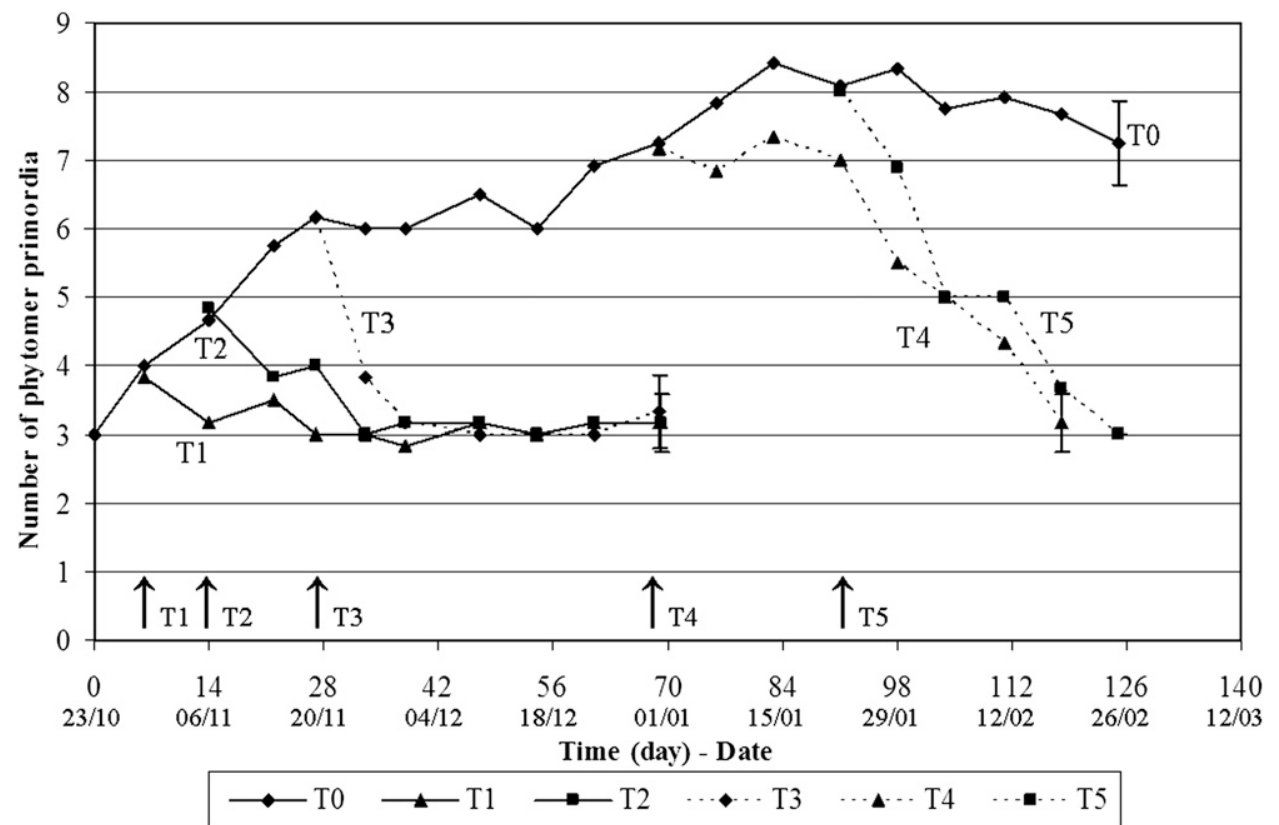

Fig. 4. Evolution of the number of phytomer primordia in the bud for treatments T0 (control plants) and T1 to T5. The plants of treatments T1, T2, T3, T4, and T5 were transferred from climatic conditions favorable to floral transformation (green house) to climatic conditions favorable to vegetative growth (climatic chamber) when the number of phytomer primordia in the bud was equal to four, five, six, seven, and eight, respectively. 
young plants resulting from asexual and clonal reproduction using mother microplant culture (Galopin et al., 1996).

Under climatic conditions favorable to floral induction in treatment T0 (Bailey, 1989), the phytomer primordial stock in the bud increased from three to eight in $83 \mathrm{~d}$ with an evolution of the real plastochron. It was equal to $8.6 \mathrm{~d}$ until the 27 th day. This value is close to $7.7 \mathrm{~d}$, which is the real plastochron observed during the organogenesis phase of vegetative growth (A) (Galopin et al., 2010). During this period, meristematic functioning was therefore slightly modified. In contrast, as of the 28th day, the plastochron considerably increased, revealing a modification of the functioning of the vegetative meristem before floral evocation (Phase $\mathrm{B}_{2}$ ). Phase $\mathrm{B}_{1}$ was followed by Phases $\mathrm{B}_{2}$ and $\mathrm{B}_{3}$ with the appearance of the first inflorescential primordia as of Day 104. The application of climatic conditions favorable to vegetative growth during Phase $B_{1}$ with treatments T1 to T5 led to a decrease of the number of phytomer primordia in the bud until it reached three, characteristic of the growing vegetative bud. In this case, the most external phytomer primordia in the bud lengthened to form visible phytomers, leading to elongation of the stem. Phase $B_{2}$ was not reached and the floral transformation process came to an end.

This experiment therefore shows that a change in climatic conditions during Phase $\mathrm{B}_{1}$ when the number of phytomer primordia in the bud is less than or equal to eight leads to the end of the floral transformation process and a resumption of vegetative development. However, is this really proof of floral reversal in H. macrophylla as was demonstrated in Impatiens balsamina and Glycine (Hempel et al., 2000; Washburn and Thomas, 2000) in which the meristem can once again form vegetative organs after the formation of floral organs? We observed that climatic conditions favorable to vegetative growth (temperature greater than $18{ }^{\circ} \mathrm{C}$; photoperiod $=16 \mathrm{~h}$ ) did not interrupt the floral transformation process in the case of $H$. macrophylla plants that had reached floral Stage 2 (beginning of Phase $\mathrm{B}_{3}$ ). After $57 \mathrm{~d}$ of culture, the number of phytomer primordia in the bud was equal to $6.92 \pm 0.51$ and the floral stage had evolved from Stage 2 to Stage 5/6. Therefore, no evidence of floral reversal in $H$. macrophylla could be observed, only a possible interruption of floral induction in Phase $B_{1}$.

Our study clearly shows that climatic conditions favorable to floral transformation must be continuously applied without interruption during the entire floral induction phase $\left(\mathrm{B}_{1}\right)$ to ensure the formation of the floral bud in Hydrangea macrophylla. The number of phytomer primordia present in the bud is a good indicator for monitoring development in Phase $\mathrm{B}_{1}$. This indicator can be used during production to predict plant development in relation to climatic conditions.

\section{Literature Cited}

Bailey, D.A. 1989. Hydrangea production. Growers Handbook Series. Vol. 3. Timber Press, Portland, OR.

Bailey, D.A. and T.C. Weiler. 1984. Stimulation of inflorescence expansion in florists' hydrangea. J. Amer. Soc. Hort. Sci. 109:792-794.

Barlow, P.W. 1989. Meristems, metamers and modules and the development of shoot and root systems. Bot. J. Linn. Soc. 100:255-279.

Chaikiattiyos, S., C.M. Menzel, and T.S. Rasmussen. 1994. Floral induction in tropical fruit trees: Effects of temperature and water supply. J. Hort. Sci. 69:397-415.

Fuchigami, L.H. and M. Wisniewski. 1997. Quantifying bud dormancy: Physiological approaches. HortScience 32:618-623.

Galopin, G. 1995. Biologie du développement d'Hydrangea macrophylla. Caractérisation du potentiel morphogénétique et maîtrise de ses expressions par les conditions de culture. Thèse de Doct. Phys. Vég., Univ. Clermont-Ferrand.

Galopin, G., F. Beaujard, and M. Gendraud. 1996 Intensive production of juvenile cuttings by mother microplant culture in Hydrangea macrophylla 'Leuchtfeuer'. Can. J. Bot. 74:561567.
Galopin, G., S. Codarin, J.D. Viemont, and P. Morel. 2008. Architectural development of inflorescence in Hydrangea macrophylla cv Hermann Dienemann. HortScience 43:361365 .

Galopin, G., J.C. Mauget, and P. Morel. 2010. Morphogenetic analysis of the phenotypic variability of the architectural unit of Hydrangea macrophylla. Ann. For. Sci. 67:309-320.

Guo, Z., M. Goi, M. Tanaka, and S. Fukai. 1995 Effects of temperature and photoperiod on the bud formation of hydrangea. Technical bulletin of the Faculty of Agriculture. Kagawa University 47:23-31.

Hempel, F.D., D.R. Welch, and L.J. Feldam. 2000. Floral induction and determination: Where is flowering controlled? Plant Sci. 5:17-21.

Morita, M., S. Iwamoto, and H. Higuchi. 1980. Interrelated effect between thermo- and photoperiodism on growth and development of ornamental woody plants. V. Modification of photoperiodic response to temperature treatment in hydrangea. J. Jpn. Soc. Hort. Sci. 48:488-494

O'Hare, T.J. 2004. Impact of root and shoot temperature on bud dormancy and floral induction in lychee (Litchi chinensis Sonn.). Sci. Hort. 99:21-28.

Post, K. 1942. Effects of day length and temperature on growth and flowering of some florist crops. Cornell University Agricultural Experiment Station, Ithaca, New York. 787: 45-52.

Shanks, J.B., H.G. Mityga, and L.W. Douglass. 1986. Photoperiodic responses of hydrangea. J. Amer. Soc. Hort. Sci. 111:545-548.

Uemachi, T. and T. Nishio. 2000. Inflorescence development in Hydrangea macrophylla. Acta Hort. 515:265-271.

Wallerstein, L. 1981. The nature of dormancy in Hydrangea macrophylla. Hassadeh 61:18461848.

Washburn, C.F. and J.F. Thomas. 2000. Reversion of flowering in Glycine max (Fabaceae). Amer. J. Bot. 87:1425-1438.

White, J. 1979. The plant as a metapopulation. Annu. Rev. Ecol. Syst. 10:109-145.

Zhou, T.S. and N. Hara. 1988. Development of shoot in Hydrangea macrophylla. I. Terminal and axillary buds. Bot. Mag. Tokyo 101:281291. 